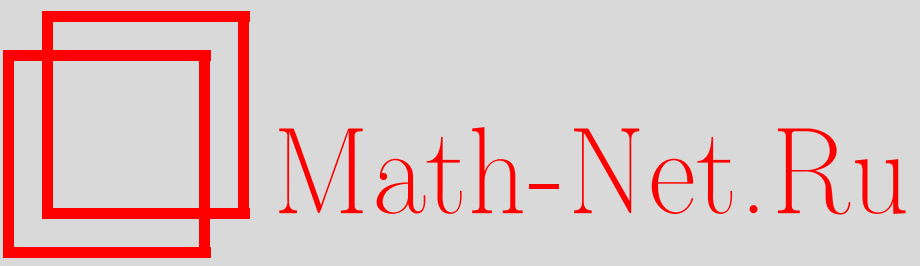

В. В. Мхитарян, Магнетик Годена и волновые функции Бете вне массовой поверхности, ТМФ, 1997, том 113, номер 1, 13-28

DOI: https://doi.org/10.4213/tmf1061

Использование Общероссийского математического портала Math-Net.Ru подразумевает, что вы прочитали и согласны с пользовательским соглашением

http://www.mathnet.ru/rus/agreement

Параметры загрузки:

IP : 54.196 .121 .252

26 апреля 2023 г., 15:25:41

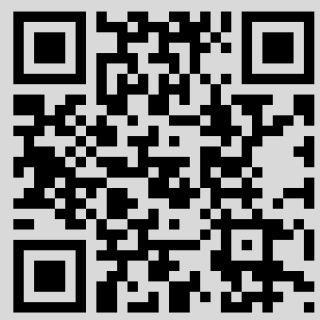




\section{МАГНЕТИК ГОДЕНА И ВОЛНОВЫЕ ФУНКЦИИ БЕТЕ ВНЕ МАССОВОЙ ПОВЕРХНОСТИ}

\footnotetext{
Доказано обобщенное уравнение Бете (уравнение анзаца Бете вне массовой поверхности) для магнетика Годена в случае широкого класса простых комплексных алгебр Ли.
}

В теории точно решаемых моделей рассматриваются задачи, относящиеся к диагонализации класса гамильтонианов, квадратичных по спиновым переменным. Речь идет о нахождении собственных состояний и собственных значений гамильтонианов

$$
H_{n}=\sum_{m} \mathcal{W}_{n m} \cdot \mathbf{S}_{n} \otimes \mathbf{S}_{m}
$$

где $\mathcal{W}$ - константы, а $\mathbf{S}_{n}$ - генераторы простой алгебры Ли.

В простейшем случае алгебры Ли $s l(2)$ при определенном выборе констант $\mathcal{W}$ задача диагонализации (1) решена в [1]. С помошью так называемого иерархического анзаца Бете можно построить собственные значения (1) для представлений некоторых конкретных алгебр Ли [2-4]. Этот метод, как правило, не дает явной реализации собственных векторов (1), которые в отдельных случаях могут быть полезными $[5,6]$.

Авторами работы [7] была предложена явная конструкция (так называемое уравнение анзаца Бете вне массовой поверхности), которая дает решение таких задач для любых простых алгебр Ли. Более того, с помощью этой конструкции можно найти конкретную реализацию решений уравнений Книжника-Замолодчикова [8] в некоторых конформных теориях [9]. Этот результат впоследствии был доказан в работе [10] с помошью бозонизации (модуль Вакимото) аффиинных алгебр $\widehat{\mathcal{G}}$.

В настоящей работе представлено доказательство результата [7] для всех простых комплексных алгебр, кроме $E_{8}, G_{2}$ и $F_{4}$. Приведенное здесь доказательство в корне отличается от [10] и приближается к методу иерархического анзаца Бете.

Ниже (в пункте 1) подробно представлена конструкция уравнения анзаца Бете вне массовой поверхности. Пункт 2 является подготовительным к пункту 3 , где и приведено окончательное доказательство.

1. Допустим, что $R^{0 i}(\mu, \eta)$ и $R^{00}(\mu, \eta)$ - решения уравнения Янга-Бакстера для простой алгебры Ли $\mathcal{G}$ с глобальным спектральным параметром $\mu$ и параметром $\eta$, который представляет собой меру отклонения от классического предела (так называемый параметр типа постоянной Планка). Это значит, что $R^{00}$ и $R^{0 i}$ действуют в пространствах

*Ереванский физический институт, Ереван, Армения. E-mail: vgho@lx2.yerphi.am 
$V^{0} \otimes V^{0}$ и $V^{0} \otimes V^{i}$, соответственно, где $V^{0}$ - пространство фундаментального представления $\pi_{0}$, a $V^{i}$ - пространство произвольного представления $\pi_{i}$ алгебры $\mathcal{G}$, и удовлетворяют соотношению Янга-Бакстера

$$
R^{00}(\mu-\lambda) R^{0 i}(\mu) R^{0 j}(\lambda)=R^{0 j}(\lambda) R^{0 i}(\mu) R^{00}(\mu-\lambda)
$$

Оно дает возможность ввести трансфер-матрицу (матрицу переноса)

$$
T\left(\mu,\left\{Z_{a}\right\}\right)=\operatorname{tr}_{0} \prod_{a=1}^{N} R^{0 a}\left(\mu-Z_{a}\right)
$$

с локальными параметрами беспорядка $Z_{1}, \ldots, Z_{N}$. Для этой матришы имеет место соотношение

$$
\left[T\left(\lambda,\left\{Z_{a}\right\}\right), T\left(\mu,\left\{Z_{a}\right\}\right)\right]=0
$$

при любых $\mu$ и $\lambda$. Матрица переноса действует в пространстве $N$-кратного тензорного произведения $V^{1} \otimes \cdots \otimes V^{N}$ и благодаря (2) является производящей функцией для интегралов движения [11]. $R$-матрица имеет квазиклассическое разложение

$$
R^{a b}(\mu)=I^{a} \otimes I^{b}+\eta r_{a b}(\mu)+O\left(\eta^{2}\right)
$$

где $r_{a b}$ - классическая $r$-матрища, удовлетворяющая классическому уравнению ЯнгаБакстера [11]

$$
\left[r_{a b}(\lambda-\mu), r_{a c}(\lambda)+r_{b c}(\mu)\right]+\left[r_{a c}(\lambda), r_{b c}(\mu)\right]=0,
$$

здесь буквами $a, b, c$ обозначены три различных представления алгебры $\mathcal{G}$.

Будем рассматривать случай рационального решения $r_{a b}=\left(t^{a}, t^{b}\right) / \mu$, где $\left(t^{a}, t^{b}\right)$ - форма Киллинга для генераторов алгебры Ли в представлениях $V^{a}, V^{b}:\left(t^{a}, t^{b}\right)=$ $K^{\alpha \beta} t_{\alpha}^{a} \otimes t_{\beta}^{b}$, где $\left\{t_{\alpha}\right\}$ - генераторы, а $K^{\alpha \beta}$ - обратная к матрище Картана $K_{\alpha \beta}$ алгебры $\mathcal{G}$. В этом случае имеем

$$
\begin{aligned}
T\left(\mu,\left\{Z_{a}\right\}\right) & =\text { const } \cdot I+\eta^{2} \sum_{a=1}^{N} \frac{H^{a}}{\mu-Z_{a}}+O\left(\eta^{3}\right), \\
H^{a} & =\sum_{b \neq a}^{N} \frac{\left(t^{a}, t^{b}\right)}{Z_{a}-Z_{b}} .
\end{aligned}
$$

Из (2) следует коммутативность

$$
\left[H^{a}, H^{b}\right]=0, \quad a, b=1, \ldots, N .
$$

Гамильтонианы (3) диагонализуются одновременно и задают магнетик Годена. Соотношение (4) приводит к тому, что следуюший класс гамильтонианов типа Гейзенберга

$$
H=\sum_{n<m} \mathcal{W}_{n m}\left(t^{n}, t^{m}\right) \equiv \sum_{a} \epsilon_{a} H_{a}
$$

вполне интегрируем, если коэффициенты имеют вид $\mathcal{W}_{n m}=\left(\epsilon_{n}-\epsilon_{m}\right) /\left(Z_{n}-Z_{m}\right)$. 
Можно построить также "классическую" матрицу переноса $t\left(\mu,\left\{Z_{a}\right\}\right)$ : обозначим

$$
l\left(\mu,\left\{Z_{a}\right\}\right)=\sum_{a=1}^{N} r_{0 a}\left(\mu-Z_{a}\right)
$$

тогда

$$
t\left(\mu,\left\{Z_{a}\right\}\right)=\frac{1}{2} \operatorname{tr}_{0} l^{2}\left(\mu,\left\{Z_{a}\right\}\right), \quad \operatorname{res}_{\mu=Z_{a}} t(\mu)=H^{a} .
$$

Пусть $e_{ \pm \alpha}, h_{\alpha}$ - генераторы $\mathcal{G}$ в базисе Картана-Вейля, соответствуюшие простому корню $\alpha$. Если П - множество простых корней $\mathcal{G}$, то корневые векторы $e_{\beta}, \beta \notin \Pi$, дополняют $\left\{e_{ \pm \alpha}, h_{\alpha}, \alpha \in \Pi\right\}$ до базиса Картана-Вейля. Операторы, соответствуюшие этим генераторам в конкретном представлении (скажем, в $V^{a}$ ), обозначим через $e_{ \pm \alpha}^{a}, h_{\alpha}^{a}$.

Допустим, что $A_{\Pi}=\left\{\alpha_{1}, \ldots, \alpha_{m}\right\}$ - некоторое множество простых корней (при этом один и тот же корень может входить в $A_{\Pi}$ несколько раз). Сопоставим каждому простому корню $\alpha_{i} \in A_{\Pi}$ комплекснозначный параметр $\mu_{i}, \mu_{i} \leftrightarrow \alpha_{i}, \mu_{i} \in \mathbf{C}$, и обозначим через $A$ множество всех пар $\left[\alpha_{i} ; \mu_{i}\right]: A=\left\{\left[\alpha_{i} ; \mu_{i}\right], \alpha_{i} \in A_{\Pi}\right\}$, а через $K$ - подмножество $A$ :

$$
K=\left\{\left[\alpha_{i}^{K} ; \mu_{i}^{K}\right],\left[\alpha_{i}^{K} ; \mu_{i}^{K}\right] \equiv\left[\alpha_{l_{i}} ; \mu_{l_{i}}\right] \in A, i=1, \ldots,|K| \leq m\right\} .
$$

Следуя [7], определим оператор

$$
X(K, a)=\sum_{p \in S_{|K|}} \frac{e_{-\alpha_{p(1)}^{K}}^{a} \ldots e_{-\alpha_{p(|K|)}^{K}}^{a}}{\left(\mu_{p(1)}^{K}-\mu_{p(2)}^{K}\right) \ldots\left(\mu_{p(|K|)}^{K}-Z_{a}\right)},
$$

где сумма проводится по всей группе перестановок $S_{|K|}$. С помощью этого оператора можно построить вектор в $V^{1} \otimes \cdots \otimes V^{N}$ :

$$
\varphi(A)=\sum_{\substack{\left(K_{1}, \ldots, K_{N}\right) \\ \bigcup_{a} K_{a}=A \\ K_{a} \cap K_{b}=\varnothing}} \prod_{a=1}^{N} \otimes X\left(K_{a}, a\right)\left|\lambda^{a}\right\rangle,
$$

где сумма берется по всем возможным попарно непересекаюшимся разбиениям $A$, а $\left|\lambda^{a}\right\rangle$ - старший вектор неприводимого представления $\pi_{a}$ в пространстве $V^{a}$.

Далее для удобства обозначений попарно непересекаюшиеся множества мы будем брать в круглые скобки, т.е. записывать $\left(K_{1}, \ldots, K_{L}\right)$, когда для любой пары индексов $a, b$ из $1, \ldots, L$ выполнены соотношения $K_{a} \cap K_{b}=\varnothing$.

Теорема. Вектор $\varphi(A)$ удовлетворяет соотношению

$$
H^{a} \varphi(A)=h^{a} \varphi(A)+\sum_{j=1}^{m} f_{\mu_{j}} \varphi_{\mu_{j}}^{a}(A),
$$


¿де

$$
\begin{aligned}
h^{a} & =\sum_{b \neq a}^{N} \frac{\left(\lambda^{a}, \lambda^{b}\right)}{Z_{a}-Z_{b}}-\sum_{j=1}^{m} \frac{\left(\alpha_{j}, \lambda^{a}\right)}{Z_{a}-\mu_{j}}, \\
f_{\mu_{j}} & =\sum_{i \neq j}^{m} \frac{\left(\alpha_{j}, \alpha_{i}\right)}{\mu_{j}-\mu_{i}}-\sum_{a=1}^{N} \frac{\left(\alpha_{j}, \lambda^{a}\right)}{\mu_{j}-Z_{a}}, \\
\varphi_{\mu_{j}}^{a}(A) & =\sum_{\substack{\left(K_{1}, \ldots, K_{N}\right) \\
\bigcup_{a} K_{a}=A \\
\left[\alpha_{j} ; \mu_{j}\right] \in K_{a}}} \prod_{b=1}^{N} \otimes X\left(K_{b}, b\right)\left|\lambda^{b}\right\rangle
\end{aligned}
$$

при любом А, где сумма (9) - это подсумма суммы (5) с разбиениями, в которых $\left[\alpha_{j} ; \mu_{j}\right] \in K_{a}[7]$.

С помошью этой теоремы можно диагонализовать гамильтонианы (3). Действительно, если наложить на $\mu_{i}$ связи (так называемое условие массовой поверхности)

$$
f_{\mu_{j}}=0, \quad\left[\alpha_{j} ; \mu_{j}\right] \in A,
$$

то гамильтонианы (3) станут диагональными. Соотношения (10) фактически являются уравнениями Бете.

В оставшейся части работы мы будем доказывать это утверждение для серий простых алгебр Ли

$$
A_{n}, \quad n \geq 1 ; \quad B_{n}, \quad n \geq 2 ; \quad C_{n}, \quad n \geq 3 ; \quad D_{n}, \quad n \geq 4 ; \quad E_{6} ; \quad E_{7} .
$$

2. Пусть теперь алгебра Ли $\mathcal{G}$ - одна из алгебр (11). В пространстве представления $V^{a}$ алгебры Ли $\mathcal{G}$ определим оператор

$$
Y^{\alpha}(K, a)=\sum_{r \in S_{|K|}} \frac{\left[e_{-\alpha_{r(1)}^{K}}^{a}\left[\ldots\left[e_{-\alpha_{r|K|}^{K}}^{a}, e_{-\alpha}^{a}\right]\right] \cdots\right]}{\left(\mu_{r(1)}^{K}-\mu_{r(2)}^{K}\right) \ldots\left(\mu_{r|K|}^{K}-\mu\right)\left(\mu-Z_{a}\right)}, \quad[\alpha ; \mu] \in A, \quad[\alpha ; \mu] \notin K .
$$

Оказывается, что имеет место соотношение

$$
\begin{aligned}
\varphi(A)= & \sum_{\substack{\left(K_{0}, K_{1}, \ldots, K_{N}\right) \\
\bigcup_{i} K_{i}=A \backslash[\alpha ; \mu]}} \sum_{b=1}^{N} \prod_{a=1}^{b-1} \otimes X\left(K_{a}, a\right)\left|\lambda^{a}\right\rangle \otimes Y^{\alpha}\left(K_{0}, b\right) X\left(K_{b}, b\right)\left|\lambda^{b}\right\rangle \times \\
& \times \prod_{a=b+1}^{N} \otimes X\left(K_{a}, a\right)\left|\lambda^{a}\right\rangle, \quad i, j=0,1, \ldots, N,
\end{aligned}
$$

где сумма берется по всем возможным $N+1$ разбиениям множества $A \backslash[\alpha ; \mu]$. 
Действительно,

$$
\begin{aligned}
& \sum_{\substack{\left(K_{0}, K_{1}, \ldots, K_{N}\right) \\
\bigcup_{i} K_{i}=A \backslash[\alpha ; \mu]}} \sum_{b=1}^{N} \prod_{a=1}^{b-1} \otimes X\left(K_{a}, a\right)\left|\lambda^{a}\right\rangle \otimes Y^{\alpha}\left(K_{0}, b\right) X\left(K_{b}, b\right)\left|\lambda^{b}\right\rangle \times \\
& \times \prod_{a=b+1}^{N} \otimes X\left(K_{a}, a\right)\left|\lambda^{a}\right\rangle= \\
& =\sum_{b=1}^{N} \sum_{\substack{\left(K_{1}, \ldots, \widehat{K}_{b}, \ldots, K_{N}\right) \\
\cup_{a \neq b} K_{a}=A \backslash(K \cup[\alpha ; \mu])}} \sum_{\substack{\left(K_{b}, K_{0}\right) \\
K_{b} \cup K_{0}=K \subset A \backslash[\alpha ; \mu]}} \prod_{a=1}^{b-1} \otimes X\left(K_{a}, a\right)\left|\lambda^{a}\right\rangle \otimes \\
& \otimes Y^{\alpha}\left(K_{0}, b\right) X\left(K_{b}, b\right)\left|\lambda^{b}\right\rangle \otimes \prod_{a=b+1}^{N} \otimes X\left(K_{a}, a\right)\left|\lambda^{a}\right\rangle= \\
& =\sum_{b=1}^{N} \sum_{\substack{\left(K_{1}, \ldots, K_{b}, \ldots, K_{N}\right) \\
\bigcup_{a} K_{a}=A \backslash[\alpha ; \mu]}} \prod_{a=1}^{b-1} \otimes X\left(K_{a}, a\right)\left|\lambda^{a}\right\rangle \otimes X\left(K_{b} \cup[\alpha ; \mu], b\right)\left|\lambda^{b}\right\rangle \times \\
& \times \prod_{a=b+1}^{N} \otimes X\left(K_{a}, a\right)\left|\lambda^{a}\right\rangle=\sum_{b=1}^{N} \sum_{\substack{\left.K_{1}, \ldots, K_{N}\right) \\
\bigcup_{a} K_{a}=A \\
[\alpha ; \mu] \in K_{b}}} \prod_{a=1}^{N} \otimes X\left(K_{a}, a\right)\left|\lambda^{a}\right\rangle= \\
& =\sum_{\substack{\left(K_{1}, \ldots, K_{N}\right) \\
\bigcup_{a} K_{a}=A}} \prod_{a=1}^{N} \otimes X\left(K_{a}, a\right)\left|\lambda^{a}\right\rangle=\varphi(A)
\end{aligned}
$$

где второе равенство следует из утверждения, доказанного в приложении, “А” означает, что соответствуюшие члены отпушены.

Для дальнейшего необходимы некоторые сведения из теории алгебр Ли и их представлений [12].

Пусть П - система простых корней алгебры Ли $\mathcal{G}$ с рангом $r$, а $\Delta$ и $\Delta^{+}-$системы всех ненулевых и положительных корней, соответственно. Тогда каждый корень $\varphi \in \Delta$ однозначно представляется в виде суммы по простым корням:

$$
\varphi=\sum n_{i} \alpha_{i}, \quad \alpha_{i} \in \Pi
$$

где $n_{i}$ - целые числа одинакового знака. Обозначим через $\Delta_{\alpha_{i}}$ подсистему корней с $n_{i} \neq$ 0 .

Оказывается, что для любой простой алгебры Ли $\mathcal{G}$ из множества (11) всегда сушествует такой простой корень $\alpha_{0} \in \Pi$, при котором в разложении (13) $n_{0}= \pm 1, \varphi \in \Delta_{\alpha_{0}}$.

Старшие веса $\chi^{i}$ фундаментальных представлений $\pi_{i}$ определяются условиями

$$
\frac{2\left(\chi_{i}, \alpha_{j}\right)}{\left(\alpha_{j}, \alpha_{j}\right)}=\delta_{i j}, \quad \alpha_{j} \in \Pi .
$$


Пусть $\chi^{0}$ - такой старший вес для $\alpha_{0}$, а $V^{0}$ - соответствуюшее пространство представления $\pi_{0},\left|\chi^{0}\right\rangle \in V^{0}$. Это представление обладает следующими свойствами:

$$
\begin{aligned}
h_{\alpha}^{0}\left|\chi^{0}\right\rangle & =\delta_{\alpha, \alpha_{0}} \frac{\left(\alpha_{0}, \alpha_{0}\right)}{2}\left|\chi^{0}\right\rangle, \quad \alpha \in \Delta, \\
e_{-\alpha}^{0}\left|\chi^{0}\right\rangle & =0, \quad \alpha \notin \Delta_{\alpha_{0}}^{+}, \\
e_{\beta}^{0} e_{-\alpha}^{0}\left|\chi^{0}\right\rangle & =\delta_{\alpha, \beta} \frac{\left(\alpha_{0}, \alpha_{0}\right)}{2}\left|\chi^{0}\right\rangle, \quad \alpha \in \Delta_{\alpha_{0}}^{+}, \quad \beta \in \Delta^{+},
\end{aligned}
$$

которые следуют из определения $\left|\chi_{0}\right\rangle$ и выбора $\alpha_{0}$.

$\mathrm{C}$ помощью представления $\pi_{0}$ определим следующие векторы в $\Omega=V^{1} \otimes \ldots$ $\cdots \otimes V^{N} \otimes V^{0}:$

$$
\psi=\sum_{\substack{\left(K_{0}, K_{1}, \ldots, K_{N}\right) \\ \cup_{a} K_{a}=A \backslash\left[\alpha_{0} ; \mu_{0}\right]}} \prod_{a=1}^{N} \otimes X\left(K_{a}, a\right)\left|\lambda^{a}\right\rangle \otimes X\left(K_{0}, \pi_{0}\right) e_{-\alpha_{0}}^{0}\left|\chi^{0}\right\rangle
$$

И

$$
\begin{aligned}
\psi_{\mu_{j}}^{\rho} & =\sum_{\substack{\left(K_{0}, K_{1}, \ldots, K_{N}\right) \\
\bigcup_{i} K_{i}=A \backslash\left[\alpha_{0} ; \mu_{0}\right] \\
\left[\alpha_{j} ; \mu_{j}\right] \in K_{\rho}}} \prod_{a=1}^{N} \otimes X\left(K_{a}, a\right)\left|\lambda^{a}\right\rangle \otimes X\left(K_{0}, \pi_{0}\right) e_{-\alpha_{0}}^{0}\left|\chi^{0}\right\rangle, \\
\rho & =0,1, \ldots, N
\end{aligned}
$$

Подобно (9) последняя сумма берется по всем тем $(N+1)$ разбиениям множества $A \backslash\left[\alpha_{0} ; \mu_{0}\right]$, для которых $\left[\alpha_{j} ; \mu_{j}\right] \in K_{\rho}$.

Обозначим через $P^{0}$ проекционный оператор в $\pi^{0}: P^{0} \equiv\left|\chi^{0}\right\rangle\left\langle\chi^{0}\right|$. В пространстве $\Omega$ определим оператор

$$
E^{0}(\mu)=P^{0} \sum_{a=1}^{N} \sum_{\alpha \in \Delta_{\alpha_{0}}^{+}} \frac{e_{-\alpha}^{a} \otimes e_{\alpha}^{0}}{\mu-Z_{a}}
$$

и докажем, что имеет место соотношение

$$
E^{0}\left(\mu_{0}\right) \psi=\frac{\left(\alpha_{0}, \alpha_{0}\right)}{2} \varphi \otimes|\chi\rangle
$$

Для этого сначала заметим, что из (14.2) следует

$$
Y^{\alpha_{0}}\left(\bar{K}_{0}, \pi_{0}\right)|\chi\rangle=X\left(\bar{K}_{0}, \pi_{0}\right) e_{-\alpha}^{0}|\chi\rangle, \quad Z_{0}=\mu_{0}
$$

Здесь и далее черта над $K$ означает, что это подмножество не содержит корня $\alpha_{0}$.

С другой стороны, $Y^{\alpha_{0}}\left(K_{0}, \pi_{0}\right)=C\left(K_{0}\right) e_{-\alpha\left(K_{0}\right)}^{0}, \alpha\left(K_{0}\right) \in \Delta_{\alpha_{0}}^{+}$, где $C\left(K_{0}\right)$ - численный коэффициент, так что для $\alpha \in \Delta_{\alpha_{0}}^{+}$из (14) получаем

$$
P^{0} e_{\alpha}^{0} X\left(K_{0}, \pi_{0}\right) e_{-\alpha_{0}}^{0}\left|\chi^{0}\right\rangle=C\left(K_{0}\right) \frac{\left(\alpha_{0}, \alpha_{0}\right)}{2} \delta_{\alpha, \alpha\left(K_{0}\right)}\left|\chi^{0}\right\rangle
$$

где $\delta_{\alpha, \beta}-$ символ Кронекера. 
Далее имеем

$$
\begin{aligned}
E^{0}\left(\mu_{0}\right) \psi= & \sum_{\substack{\left(K_{0}, K_{1}, \ldots, K_{N}\right) \\
\cup_{a} K_{a}=A \backslash\left[\alpha_{0} ; \mu_{0}\right]}} \sum_{a=1}^{N} \sum_{\alpha \in \Delta_{\alpha_{0}}^{+}} \frac{e_{-\alpha}^{a}}{\mu_{0}-Z_{a}} \times \\
& \times \prod_{b=1}^{N} \otimes X\left(K_{b}, b\right)\left|\lambda^{b}\right\rangle \otimes P^{0} e_{\alpha}^{0} X\left(K_{0}, \pi_{0}\right) e_{-\alpha_{0}}^{0}\left|\chi^{0}\right\rangle= \\
= & \frac{\left(\alpha_{0}, \alpha_{0}\right)}{2} \sum_{\left(K_{0}, K_{1}, \ldots, K_{N}\right)} \sum_{a=1}^{N} \sum_{\alpha \in \Delta_{\alpha_{0}}^{+}} \frac{C\left(K_{0}\right) e_{-\alpha\left(K_{0}\right)}^{a} \times}{\mu_{0}-Z_{a}} \times \\
& \times \prod_{b=1}^{N} \otimes X\left(K_{b}, b\right)\left|\lambda^{b}\right\rangle \otimes\left|\chi^{0}\right\rangle= \\
= & \frac{\left(\alpha_{0}, \alpha_{0}\right)}{2} \sum_{\left(K_{0}, K_{1}, \ldots, K_{N}\right)}^{\left(K_{0} ; \mu_{0}\right]} \sum_{a=1}^{N} Y^{\alpha_{0}}\left(K_{0}, a\right) \prod_{b=1}^{N} \otimes X\left(K_{b}, b\right)\left|\lambda^{b}\right\rangle \otimes\left|\chi^{0}\right\rangle= \\
= & \frac{\left(\alpha_{0}, \alpha_{0}\right)}{2} \varphi \otimes\left|\chi^{0}\right\rangle, K_{a}=A\left[\alpha_{0} ; \mu_{0}\right]
\end{aligned}
$$

где последнее равенство следует из (12).

Если в $\Omega$ определить оператор

$$
E^{0 \mid a}(\mu)=P^{0} \sum_{\alpha \in \Delta_{\alpha_{0}}^{+}} \frac{e_{-\alpha}^{a} \otimes e_{\alpha}^{0}}{\mu-Z_{a}},
$$

то таким же образом можно убедиться в справедливости следуюших соотношений:

$$
\begin{aligned}
& E^{0}\left(\mu_{0}\right) \psi_{\mu_{j}}^{a}+E^{0 \mid a}\left(\mu_{0}\right) \psi_{\mu_{j}}^{0}=\frac{\left(\alpha_{0}, \alpha_{0}\right)}{2} \varphi_{\mu_{j}}^{a} \otimes|\chi\rangle, \quad a=1, \ldots, N, \\
& E^{0 \mid a}\left(\mu_{0}\right) \psi=\frac{\left(\alpha_{0}, \alpha_{0}\right)}{2} \varphi_{\mu_{0}}^{a} \otimes|\chi\rangle .
\end{aligned}
$$

При этом важно отметить, что для выполнения (18), (20) и (21) выбор $\alpha_{0}$ оказывается существенным.

Заметим, что кратность $\alpha_{0} \in A_{\Pi}$ с самого начала произвольна, так что можно несколько обобшить соотношения (18) и (20). Действительно, пусть кратность $\alpha_{0}$ в $A$ равна $p$. Отметим это явно посредством добавления индекса $p$ к $A: A \rightarrow A_{p}$. Переобозначим параметры $\mu$, соответствуюшие $\alpha_{0}$, через $\mu_{0 i}, i=1, \ldots, p$, и в пространстве

$$
\Omega^{p}=V^{1} \otimes \cdots \otimes V^{N} \otimes V^{01} \otimes \cdots \otimes V^{0 p}, \quad V_{0 i} \equiv V^{0},
$$

определим операторы $E^{0_{i}}(\mu), E^{0_{i} \mid a}(\mu)$, подобные операторам (17), (19), соответственно,

$$
\begin{aligned}
E^{0_{i}}(\mu) & =P^{0_{i}} \sum_{\rho=1}^{N+i-1} \sum_{\alpha \in \Delta_{\alpha_{0}}^{+}} \frac{e_{-\alpha}^{\rho} \otimes e_{\alpha}^{0_{i}}}{\mu-Z_{\rho}}, \quad i=1, \ldots, p, \\
E^{0_{i} \mid a}(\mu) & =P^{0_{i}} \sum_{\alpha \in \Delta_{\alpha_{0}}^{+}} \frac{e_{-\alpha}^{a} \otimes e_{\alpha}^{0_{i}}}{\mu-Z_{a}}, \quad a=1, \ldots, N,
\end{aligned}
$$


и векторы $\psi^{p}, \psi_{\mu_{j}}^{p \mid \rho}$, аналогичные векторам (15) и (16):

$$
\begin{aligned}
& \psi^{p}=\sum_{\substack{\left(K_{01}, \ldots, K_{0 p}, K_{1}, \ldots, K_{N}\right) \\
\bigcup_{\sigma} K_{\sigma}=\bar{A}}} \prod_{a=1}^{N} \otimes X\left(K_{a}, a\right)\left|\lambda^{a}\right\rangle \prod_{i=1}^{p} \otimes X\left(K_{0_{i}}, 0_{i}\right) e_{-\alpha_{0}}^{0_{i}}\left|\chi^{0_{i}}\right\rangle, \\
& \psi_{\mu_{j}}^{p \mid \rho}=\sum_{\substack{\left(K_{01}, \ldots, K_{0 p}, K_{1}, \ldots, K_{N}\right) \\
\bigcup_{\sigma} K_{\sigma}=\bar{A} \\
\left[\alpha_{j} ; \mu_{j}\right] \in K_{\rho}}} \prod_{a=1}^{N} \otimes X\left(K_{a}, a\right)\left|\lambda^{a}\right\rangle \prod_{i=1}^{p} \otimes X\left(K_{0_{i}}, 0_{i}\right) e_{-\alpha_{0}}^{0_{i}}\left|\chi^{0_{i}}\right\rangle \\
& \rho=1, \ldots, N, 0_{1}, \ldots, 0_{p} .
\end{aligned}
$$

Здесь $\bar{A}$ - такое множество, которое получается из множества $A$ удалением всех элементов $\left\{\left[\alpha_{0} ; \mu_{0_{i}}\right]\right\}_{i=1}^{p}$. Последняя сумма подобно суммам в формулах (9) и (16) берется по всем тем $(N+p)$ разбиениям множества $\bar{A}$, для которых $\left[\alpha_{j} ; \mu_{j}\right] \in K_{\rho}$.

Обобщая (18), (20) и (21), можно получить следующие соотношения:

$$
\begin{aligned}
& \prod_{i=1}^{p} E^{0_{i}}\left(\mu_{0_{i}}\right) \psi^{p}=\left[\frac{\left(\alpha_{0}, \alpha_{0}\right)}{2}\right]^{p} \varphi \otimes \prod_{i=1}^{p} \otimes\left|\chi^{0_{i}}\right\rangle \\
& \prod_{i=1}^{p} E^{0_{i}}\left(\mu_{0_{i}}\right) \psi_{\mu_{j}}^{p \mid a}+\sum_{k=1}^{p} E^{0_{1}}\left(\mu_{0_{1}}\right) \ldots E^{0_{k} \mid a}\left(\mu_{0_{k}}\right) \ldots E^{0_{p}}\left(\mu_{0_{p}}\right) \psi_{\mu_{j}}^{p \mid 0_{k}}= \\
& \quad=\left[\frac{\left(\alpha_{0}, \alpha_{0}\right)}{2}\right]^{p} \varphi_{\mu_{j}}^{a} \otimes \prod_{i=1}^{p} \otimes\left|\chi^{0_{i}}\right\rangle, \quad\left[\alpha_{j} ; \mu_{j}\right] \in \bar{A}, \\
& E^{0_{1}}\left(\mu_{0_{1}}\right) \ldots E^{0_{k}} \mid a\left(\mu_{0_{k}}\right) \ldots E^{0_{p}}\left(\mu_{0_{p}}\right) \psi^{p}= \\
& \quad=\left[\frac{\left(\alpha_{0}, \alpha_{0}\right)}{2}\right]^{p} \varphi_{\mu_{0_{k}}}^{a} \prod_{i=1}^{p} \otimes\left|\chi^{0_{i}}\right\rangle, \quad k=1, \ldots, p .
\end{aligned}
$$

Для дальнейшего важно заметить, что

$$
\begin{aligned}
& P^{0_{i}} e_{\alpha_{0}}^{0_{i}}\left(e_{-\alpha_{1}}^{0_{i}} \otimes e_{\alpha_{1}}^{0_{k_{1}}} \otimes \cdots \otimes e_{-\alpha_{n}}^{0_{i}} \otimes e_{\alpha_{n}}^{0_{k_{n}}}\right) \psi^{p}=0 \\
& i<k_{1}<\cdots<k_{n}, \quad \alpha_{0}, \ldots, \alpha_{n} \in \Delta_{\alpha_{0}}^{+} .
\end{aligned}
$$

Это приводит к тому, что в (24) все операторы $E$ можно заменить следующими операторами $\bar{E}$ :

$$
\begin{aligned}
& E^{0_{i}}(\mu) \rightarrow \bar{E}^{0_{i}}(\mu)=P^{0_{i}} \sum_{b=1}^{N} \sum_{\alpha \in \Delta_{\alpha_{0}}^{+}} \frac{e_{-\alpha}^{b} \otimes e_{\alpha}^{0_{i}}}{\mu-Z_{b}}, \quad i=1, \ldots, p, \\
& E^{0_{i} \mid a}(\mu) \rightarrow \bar{E}^{0_{i} \mid a}(\mu) \equiv E^{0_{i} \mid a}(\mu) .
\end{aligned}
$$

Из коммутативности

$$
\left[e_{ \pm \alpha_{1}}, e_{ \pm \alpha_{2}}\right]=0, \quad \alpha_{1}, \alpha_{2} \in \Delta_{\alpha_{0}}^{+}
$$


вытекает, что операторы $\bar{E}^{0_{i}}(\mu)$ с разными $i$ коммутируют между собой и с операторами $\bar{E}^{0_{j} \mid a}(\mu)$.

3. Будем доказывать утверждение с помощью математической индукции по рангу алгебры. В данном случае нам предстоит пройти следуюшие два этапа:

а) проверить справедливость утверждения для алгебр Ли ранга 1 ;

б) допуская его справедливость для всех алгебр Ли ранга $r-1$, доказывать корректность утверждения для алгебр Ли с рангом $r$.

Докажем пункт "a". Принимая во внимание равенство

$$
\frac{1}{\mu_{1}-Z} \cdots \frac{1}{\mu_{m}-Z}=\sum_{p \in S(m)} \frac{1}{\left(\mu_{p(1)}-\mu_{p(2)}\right) \ldots\left(\mu_{p(m)}-Z\right)}
$$

и следуя [1, пункт 13.1.2], нетрудно убедиться в справедливости соотношений (6)-(9) для алгебры Ли $s l(2)$. Это доказывает утверждение нашей теоремы для алгебр Ли ранга 1 , т.к. $s l(2)$ - единственная простая комплексная алгебра Ли единичного ранга.

Приступим к рассмотрению пункта "б". Пусть $\mathcal{G}$-произвольная простая алгебра Ли ранга $r$ из (11), a $\left\{h_{\alpha}, e_{\beta} ; \alpha \in \Pi, \beta \in \Delta\right\}$ - конкретный базис Картана-Вейля алгебры Ли $\mathcal{G}$.

Перейдем к рассмотрению несколько другого базиса $\left\{\hat{h}_{\alpha}, \hat{e}_{\beta} ; \alpha \in \Pi, \beta \in \Delta\right\}$ алгебры $\mathcal{G}$, определяя его следующим образом:

$$
\begin{aligned}
\hat{h}_{\alpha_{0}} & =\sum_{\delta \in \Pi} M^{\alpha_{0} \delta} h_{\delta}, \\
\hat{h}_{\alpha} & =h_{\alpha}, \quad \alpha \neq \alpha_{0}, \\
\hat{e}_{ \pm \beta} & =e_{ \pm \beta}, \quad \beta \in \Delta,
\end{aligned}
$$

где $M^{\alpha \beta}$ - обратная к матрице $M_{\alpha \beta}=\left(h_{\alpha}, h_{\beta}\right)$. Нетрудно заметить, что этот базис обладает следующими полезными свойствами:

$$
\begin{aligned}
& \left(\hat{h}_{\alpha_{0}}, \hat{h}_{\beta}\right)=0, \quad \beta \neq \alpha_{0}, \\
& {\left[\hat{h}_{\alpha_{0}}, e_{\beta}\right]=0, \quad \beta \notin \Delta_{\alpha_{0}},} \\
& {\left[\hat{h}_{\alpha_{0}}, e_{ \pm \alpha}\right]= \pm e_{ \pm \alpha}, \quad \alpha \in \Delta_{\alpha_{0}}^{+},} \\
& \widehat{M}^{\alpha_{0} \beta}=0, \quad \beta \neq \alpha_{0},
\end{aligned}
$$

где $\widehat{M}^{\alpha \beta}$ - матрица, обратная к матрице $\widehat{M}_{\alpha \beta}=\left(\hat{h}_{\alpha}, \hat{h}_{\beta}\right), \delta_{\alpha, \beta}$ - символ Кронекера.

Пользуясь уже принятыми обозначениями, в пространстве $\Omega^{p}$ определим следуюшие операторы:

$$
\begin{aligned}
H_{i}^{a} & =\sum_{q \neq a}^{N+i} \frac{\left(t^{a}, t^{q}\right)}{Z_{a}-Z_{q}}, \\
H_{i}^{0_{j}} & =\sum_{q \neq 0_{j}}^{N+i} \frac{\left(t^{0_{j}}, t^{q}\right)}{\mu_{0 i}-Z_{q}}, \quad j \leq i, \\
Z_{N+j} & \equiv \mu_{0_{j}}, \quad t^{N+j} \equiv t^{0_{j}} .
\end{aligned}
$$


Они удовлетворяют коммутационным соотношениям

$$
\left[H_{i}^{\rho}, H_{i}^{\sigma}\right]=0, \quad \rho ; \sigma=1, \ldots, N ; 0_{1}, \ldots, 0_{p}, \quad i=0, \ldots, p
$$

которые в частном случае дают

$$
H_{k-1}^{a} \otimes I^{0_{k}} H_{k}^{0_{k}}=H_{k}^{0_{k}} H_{k}^{a}+\frac{\left(t^{0_{k}} t^{a}\right)}{\mu_{0_{k}}-Z_{a}} H_{k}^{0_{k}} .
$$

Рассмотрим подалгебру алгебры $\mathcal{G}$, которая как линейное пространство порождается векторами

$$
h_{\alpha}, e_{ \pm \beta}, \quad \text { где } \alpha \in \Pi \backslash \alpha_{0}, \quad \beta \in \Delta \backslash \Delta_{\alpha_{0}} .
$$

Обозначим эту подалгебру через $\widetilde{\mathcal{G}}$, а сужение представления $\pi_{a}$ алгебры Ли $\mathcal{G}$ на подалгебру $\widetilde{\mathcal{G}}$ - через $\tilde{\pi}_{a}$. Ранг подалгебры $\widetilde{\mathcal{G}}$ равен $r-1$.

Очевидно, что весовые векторы представлений $\pi\left|\lambda^{a}\right\rangle \in V^{a}, e_{-\alpha_{0}}^{0_{i}}\left|\chi^{0_{i}}\right\rangle \in V^{0_{i}}$ играют роль старшего веса для соответствующих представлений $\tilde{\pi}$.

Схема Дынкина, соответствуюшая подалгебре $\widetilde{\mathcal{G}}$, является подсхемой для схемы Дынкина $\mathcal{G}$, поэтому

$$
\left(\widetilde{h_{\alpha}, h_{\beta}}\right)=\tau\left(h_{\alpha}, h_{\beta}\right)=\tau M_{\alpha \beta},
$$

где $\widetilde{(,)}$ - форма Киллинга подалгебры $\widetilde{\mathcal{G}}$, а $\tau$ - коэффициент пропорциональности. Рассмотрим векторы

$$
\tilde{h}_{\alpha}=1 / \tau h_{\alpha}, \quad \tilde{e}_{\beta}=1 / \sqrt{\tau} e_{\beta}, \quad \alpha \in \Pi \backslash \alpha_{0}, \quad \beta \in \Delta \backslash \Delta_{\alpha_{0}},
$$

для которых $\left(\widetilde{\tilde{e}_{\alpha}, \tilde{e}_{-\alpha}}\right)=1$. Эти векторы образуют базис Картана-Вейля алгебры $\widetilde{\mathcal{G}}$ с коммутационными соотношениями

$$
\begin{aligned}
{\left[\tilde{h}_{\alpha}, \tilde{e}_{\beta}\right] } & =\left(\widetilde{\left.\tilde{h}_{\alpha}, \tilde{h}_{\beta}\right)} \tilde{e}_{\beta},\right. \\
{\left[\tilde{e}_{\alpha}, \tilde{e}_{\beta}\right] } & =\tilde{N}_{\alpha \beta} \tilde{e}_{\alpha+\beta}, \\
{\left[\tilde{e}_{\alpha}, \tilde{e}_{-\alpha}\right] } & =\tilde{h}_{\alpha} .
\end{aligned}
$$

Это дает возможность найти значение формы Киллинга на старших векторах $\left|\lambda^{a}\right\rangle \in V^{a}$, $e_{-\alpha_{0}}^{0_{i}}\left|\chi^{0_{i}}\right\rangle \in V^{0_{i}}$ представлений $\tilde{\pi}$ :

$$
\begin{aligned}
\left(\alpha_{j}, \lambda^{\rho}\right) & =\tau\left(\widetilde{\tilde{\alpha}_{j}, \lambda^{\rho}}\right), \quad \tilde{\alpha}_{j} \equiv \tilde{h}_{\alpha_{j}}, \\
\left(\lambda^{\rho}, \lambda^{\sigma}\right) & =\left(\lambda^{\rho}, \alpha_{0}\right) \widehat{M}^{\alpha_{0}, \alpha_{0}}\left(\alpha_{0}, \lambda^{\sigma}\right)+\tau\left(\widetilde{\lambda^{\rho}, \lambda^{\sigma}}\right) \\
\rho ; \sigma & =1, \ldots, N ; 0_{1}, \ldots, 0_{p} .
\end{aligned}
$$

Принимая во внимание разложение

$$
\begin{aligned}
\left(t^{\rho}, t^{\sigma}\right)= & \sum_{\alpha \in \Delta}\left\{e_{-\alpha}^{\rho} e_{\alpha}^{\sigma}+e_{\alpha}^{\rho} e_{-\alpha}^{\sigma}\right\}+\sum_{\substack{\alpha, \beta \in \Pi \\
\alpha, \beta \neq \alpha_{0}}} \widehat{M}^{\alpha, \beta} \hat{h}_{\alpha}^{\rho} \hat{h}_{\beta}^{\sigma}+ \\
& +\widehat{M}^{\alpha_{0} \alpha_{0}} \hat{h}_{\alpha_{0}}^{\rho} \hat{h}_{\alpha_{0}}^{\sigma}+\sum_{\alpha \in \Delta_{\alpha_{0}}}\left\{e_{-\alpha}^{\rho} e_{\alpha}^{\sigma}+e_{\alpha}^{\rho} e_{-\alpha}^{\sigma}\right\} \\
\rho ; \sigma= & 1, \ldots, N ; 0_{1}, \ldots, 0_{p}
\end{aligned}
$$


мы получим

$$
\begin{aligned}
& H_{p}^{\rho}=\tau \widetilde{H}_{p}^{\rho}+\sum_{\sigma \neq \rho} \frac{\widehat{M}^{\alpha_{0} \alpha_{0}} \hat{h}_{\alpha_{0}}^{\rho} \hat{h}_{\alpha_{0}}^{\sigma}}{Z_{\rho}-Z_{\sigma}}+\sum_{\sigma \neq \rho} \sum_{\alpha \in \Delta_{\alpha_{0}}} \frac{\left\{e_{-\alpha}^{\rho} e_{\alpha}^{\sigma}+e_{\alpha}^{\rho} e_{-\alpha}^{\sigma}\right\}}{Z_{\rho}-Z_{\sigma}} \\
& \rho ; \sigma=1, \ldots, N ; 0_{1}, \ldots, 0_{p}
\end{aligned}
$$

Так как $e_{\alpha}^{\sigma} \psi^{p}=0$ при $\sigma=1, \ldots, N, \alpha \in \Delta_{\alpha_{0}}^{+}$, то из предположения индукции и из соотношений $(29),(34),(36)$ следуют соотношения

$$
\begin{aligned}
\prod_{i=1}^{p} E^{0_{i}}\left(\mu_{0_{i}}\right) H_{p}^{a} \psi^{p}\left(A_{p}\right)= & \prod_{i=1}^{p} E^{0_{i}}\left(\mu_{0_{i}}\right)\left\{\phi^{a} \psi^{p}\left(A_{p}\right)+\sum_{j=1}^{m} \tilde{f}_{\mu_{j}} \psi_{\mu_{j}}^{p \mid a}\left(A_{p}\right)\right\}+ \\
& +\prod_{j=1}^{p} E^{0_{j}}\left(\mu_{0_{j}}\right)\left(\sum_{i=1}^{p} \sum_{\alpha \in \Delta_{\alpha_{0}}} \frac{e_{-\alpha}^{a} e_{\alpha}^{0_{i}}}{Z_{a}-\mu_{0_{i}}}\right) \psi^{p}\left(A_{p}\right), \\
a= & 1, \ldots, N, \\
\prod_{i=1}^{p} E^{0_{i}}\left(\mu_{0_{i}}\right) H_{p}^{0_{k}} \psi^{p}\left(A_{p}\right)= & \prod_{i=1}^{p} E^{0_{i}}\left(\mu_{0_{i}}\right)\left\{\phi^{0_{k}} \psi^{p}\left(A_{p}\right)+\sum_{j=1}^{m} \tilde{f}_{\mu_{j}} \psi_{\mu_{j}}^{p \mid 0_{k}}\left(A_{p}\right)\right\}+ \\
& +\prod_{j=1}^{p} E^{0_{j}}\left(\mu_{0_{j}}\right)\left(\sum_{b=1}^{N} \sum_{\alpha \in \Delta_{\alpha_{0}}} \frac{e_{-\alpha}^{b} e_{0_{k}}^{0_{k}}}{\mu_{0_{k}}-Z_{b}}\right) \psi^{p}\left(A_{p}\right), \\
k= & 1, \ldots, p,
\end{aligned}
$$

здесь

$$
\begin{aligned}
\phi^{\sigma} & =\sum_{\rho \neq \sigma} \frac{\left(\lambda^{\rho}, \lambda^{\sigma}\right)}{Z_{\sigma}-Z_{\rho}}-\sum_{\left[\alpha_{j} ; \mu_{j}\right] \in \bar{A}} \frac{\left(\alpha_{j}, \lambda^{\sigma}\right)}{Z_{\sigma}-\mu_{j}} \\
\tilde{f}_{\mu_{j}} & =\sum_{\left[\alpha_{i} ; \mu_{i}\right] \neq\left[\alpha_{j} ; \mu_{j}\right]} \frac{\left(\alpha_{j}, \alpha_{i}\right)}{\mu_{j}-\mu_{i}}-\sum_{a=1}^{N} \frac{\left(\alpha_{j}, \lambda^{\rho}\right)}{\mu_{j}-Z_{\rho}}, \\
\left|\lambda^{0 i}\right\rangle & \equiv e_{-\alpha_{0}}^{0_{i}}\left|\chi^{0_{i}}\right\rangle, \quad i=1, \ldots, p, \quad \sigma ; \rho=1, \ldots, N ; 0_{1}, \ldots, 0_{p} .
\end{aligned}
$$

Умножая слева обе части соотношения (31) на $P^{0_{k}}$ и используя формулу (14), мы получим

$$
\begin{aligned}
H_{k-1}^{a} E^{0_{k}}\left(\mu_{0_{k}}\right)= & E^{0_{k}}\left(\mu_{0_{k}}\right) H_{k}^{a}+E^{0_{k} \mid a}\left(\mu_{0_{k}}\right) H_{k}^{0_{k}}+\sum_{\alpha, \beta \in \Pi} \frac{M^{\alpha \beta} h_{\alpha}^{a} \otimes h_{\beta}^{0_{k}}}{\mu_{0_{k}}-Z_{a}} E^{0_{k}}\left(\mu_{0_{k}}\right)- \\
& -\left(\sum_{a=1}^{N} \sum_{\alpha, \beta \in \Pi} \frac{M^{\alpha \beta} h_{\alpha}^{a} \otimes h_{\beta}^{0_{k}}}{\mu_{0_{k}}-Z_{a}}+\sum_{i<k} \sum_{\alpha, \beta \in \Pi} \frac{M^{\alpha \beta} h_{\alpha}^{0_{i}} \otimes h_{\beta}^{0_{k}}}{\mu_{0_{k}}-\mu_{0_{i}}}\right) \times \\
& \times E^{0_{k} \mid a}\left(\mu_{0_{k}}\right)+A^{0_{k}} P^{0_{k}}, \\
H_{k-1}^{0_{j}} E^{0_{k}}\left(\mu_{0_{k}}\right)= & E^{0_{k}}\left(\mu_{0_{k}}\right) H_{k}^{0_{j}}+\sum_{\alpha, \beta \in \Pi} \frac{M^{\alpha \beta} h_{\alpha}^{0_{k}} \otimes h_{\beta}^{0_{j}}}{\mu_{0_{k}}-\mu_{0_{j}}} E^{0_{k}}\left(\mu_{0_{k}}\right)+B^{0_{k}} P^{0_{k}}, \quad j<k,
\end{aligned}
$$


здесь $A^{0_{k}}$ и $B^{0_{k}}$ - некоторые операторы в $\Omega^{p}$. Из (14.3) и (14.1) следует справедливость следуюших соотношений:

$$
\begin{aligned}
& \sum_{\alpha, \beta \in \Pi} M^{\alpha \beta} h_{\alpha}^{0_{k}} \otimes h_{\beta}^{\rho} E^{0_{k}}\left(\mu_{0_{k}}\right)=E^{0_{k}}\left(\mu_{0_{k}}\right) \hat{h}_{\alpha_{0}}^{\rho}, \\
& \sum_{\alpha, \beta \in \Pi} M^{\alpha \beta} h_{\alpha}^{0_{k}} \otimes h_{\beta}^{\rho} E^{0_{k} \mid a}\left(\mu_{0_{k}}\right)=E^{0_{k} \mid a}\left(\mu_{0_{k}}\right) \hat{h}_{\alpha_{0}}^{\rho}, \\
& \rho=1, \ldots, N, 0_{1}, \ldots, 0_{p}, \quad a=1, \ldots, N .
\end{aligned}
$$

Имея в виду последние соотношения, из (40) получаем

$$
\begin{aligned}
H_{k-1}^{a} E^{0_{k}}\left(\mu_{0_{k}}\right)= & E^{0_{k}}\left(\mu_{0_{k}}\right) H_{k}^{a}+E^{0_{k} \mid a}\left(\mu_{0_{k}}\right) H_{k}^{0_{k}}+\frac{\left(\alpha_{0}, \alpha_{0}\right)}{2} E^{0_{k}}\left(\mu_{0_{k}}\right) \frac{\hat{h}_{\alpha_{0}}^{a}}{\mu_{0_{k}}-Z_{a}}- \\
& -\frac{\left(\alpha_{0}, \alpha_{0}\right)}{2} E^{0_{k} \mid a}\left(\mu_{0_{k}}\right)\left(\sum_{a=1}^{N} \frac{\hat{h}_{\alpha_{0}}^{a}}{\mu_{0_{k}}-Z_{a}}+\sum_{i<k} \frac{\hat{h}_{\alpha_{0}}^{0_{i}}}{\mu_{0_{k}}-\mu_{0_{i}}}\right)+A^{0_{k}} P^{0_{k}}, \\
H_{k-1}^{0_{i}} E^{0_{k}}\left(\mu_{0_{k}}\right)= & E^{0_{k}}\left(\mu_{0_{k}}\right) H_{k}^{0_{i}}+\frac{\left(\alpha_{0}, \alpha_{0}\right)}{2} \frac{\hat{h}_{\alpha_{0}}^{0_{i}}}{\mu_{0_{k}}-\mu_{0_{i}}} E^{0_{k}}\left(\mu_{0_{k}}\right)+B^{0_{k}} P^{0_{k}}, \quad i<k .
\end{aligned}
$$

Далее, из (24) имеем

$$
\begin{aligned}
{\left[\frac{\left(\alpha_{0}, \alpha_{0}\right)}{2}\right]^{p} H^{a} \varphi \otimes \prod_{i=1}^{p} \otimes\left|\chi^{0_{i}}\right\rangle=H^{a} \prod_{i=1}^{p} E^{0_{i}}\left(\mu_{0_{i}}\right) \psi^{p}=} \\
=\prod_{i=1}^{p} E^{0_{i}}\left(\mu_{0_{i}}\right) H_{N+p}^{a} \psi^{p}+\sum_{k=1}^{p} E^{0_{1}}\left(\mu_{0_{1}}\right) \ldots E^{0_{k-1}}\left(\mu_{0_{k-1}}\right) \times \\
\quad \times\left\{H_{N+k-1}^{a} E^{0_{k}}\left(\mu_{0_{k}}\right)-E^{0_{k}}\left(\mu_{0_{k}}\right) H_{N+k}^{a}\right\} \ldots E^{0_{p}}\left(\mu_{0_{p}}\right) \psi^{p} .
\end{aligned}
$$

Теперь необходимо разложить выражение в фигурной скобке последней суммы с помощью соотношения (41). Рассмотрим выражение $e_{\alpha_{1}}^{0_{k}} e_{-\alpha_{2}}^{0_{k}} e_{\alpha_{3}}^{0_{k}} \psi^{p}, \alpha_{i} \in \Delta_{\alpha_{0}}^{+}$. Из определения (22) следует, что

$$
e_{\alpha_{3}}^{0_{k}} \psi^{p}=\sum\left|f^{1}\right\rangle \otimes \cdots \otimes\left|f^{N}\right\rangle \otimes\left|g^{1}\right\rangle \otimes \cdots \otimes\left|\chi^{0_{k}}\right\rangle \otimes \cdots \otimes\left|g^{p}\right\rangle,
$$

где $\left|f^{a}\right\rangle \in V^{a},\left|g^{i}\right\rangle \in V^{0_{i}}$. Отсюда с помощью (14.3) получаем

$$
e_{\alpha_{1}}^{0_{k}} e_{-\alpha_{2}}^{0_{k}} e_{\alpha_{3}}^{0_{k}} \psi^{p}=\frac{\left(\alpha_{0}, \alpha_{0}\right)}{2} \delta_{\alpha_{1}, \alpha_{2}} e_{\alpha_{3}}^{0_{k}} \psi^{p}
$$

Благодаря этому с учетом (27) для последних членов в (37) и (38) мы получим

$$
\begin{aligned}
& \prod_{i=1}^{p} E^{0_{i}}\left(\mu_{0_{i}}\right) \frac{\sum_{\alpha \in \Delta_{\alpha_{0}}^{+}} e_{-\alpha}^{a} \otimes e_{\alpha}^{0_{k}}}{Z_{a}-\mu_{0_{k}}} \psi^{p}=\sum_{j>k} \prod_{i \neq j, k} \bar{E}^{0_{i}}\left(\mu_{0_{i}}\right) P^{0_{k}} P^{0_{j}} \times \\
& \quad \times \sum_{b=1}^{N} \frac{e_{-\alpha_{1}}^{b} \otimes e_{\alpha_{1}}^{0_{k}}}{\mu_{0_{k}}-Z_{b}} \frac{e_{-\alpha_{2}}^{0_{k}} \otimes e_{\alpha_{2}}^{0_{j}}}{\mu_{0_{j}}-\mu_{0_{k}}} \frac{e_{-\alpha_{3}}^{a} \otimes e_{\alpha_{3}}^{0_{k}}}{Z_{a}-\mu_{0_{k}}} \psi^{p}= \\
& =-\sum_{j>k} \frac{\left(\alpha_{0}, \alpha_{0}\right) / 2}{\mu_{0_{j}}-\mu_{0_{k}}} E^{0_{1}}\left(\mu_{0_{1}}\right) \ldots E^{0_{k} \mid a}\left(\mu_{0_{k}}\right) \ldots E^{0_{j}}\left(\mu_{0_{k}}\right) \ldots E^{0_{p}}\left(\mu_{0_{p}}\right) \psi^{p}
\end{aligned}
$$




$$
\begin{aligned}
& E^{0_{1}}\left(\mu_{0_{1}}\right) \ldots E^{0_{k} \mid a}\left(\mu_{0_{k}}\right) \ldots E^{0_{p}}\left(\mu_{0_{p}}\right) \sum_{b=1}^{N} \frac{\sum_{\alpha \in \Delta_{\alpha_{0}}^{+}} e_{-\alpha}^{b} \otimes e_{\alpha}^{0_{k}}}{\mu_{0_{k}}-Z_{b}} \psi^{p}= \\
& =\sum_{j>k} \prod_{i \neq j, k} \bar{E}^{0_{i}}\left(\mu_{0_{i}}\right) P^{0_{k}} P^{0_{j}} \frac{e_{-\alpha_{1}}^{a} \otimes e_{\alpha_{1}}^{0_{k}}}{\mu_{0_{k}}-Z_{a}} \frac{e_{-\alpha_{2}}^{0_{k}} \otimes e_{\alpha_{2}}^{0_{j}}}{\mu_{0_{j}}-\mu_{0_{k}}} \sum_{b=1}^{N} \frac{e_{-\alpha_{3}}^{b} \otimes e_{\alpha_{3}}^{0_{k}}}{\mu_{0_{k}}-Z_{b}} \psi^{p}= \\
& =\sum_{j>k} \frac{\left(\alpha_{0}, \alpha_{0}\right) / 2}{\mu_{0_{j}}-\mu_{0_{k}}} \frac{\mu_{0_{j}}-Z_{a}}{\mu_{0_{k}}-Z_{a}} \varphi_{\mu_{0_{j}}}^{a},
\end{aligned}
$$

где повторяюшиеся индексы $\alpha_{i}$ означают суммирование по всем $\alpha_{i} \in \Delta_{\alpha_{0}}^{+}$.

Используя коммутационные соотношения (29.3) и пронося операторы $\hat{h}$ направо через $E(\mu)$, с учетом (26) получаем

$$
\begin{aligned}
& \frac{\left(\alpha_{0}, \alpha_{0}\right)}{2} E^{0_{1}}\left(\mu_{0_{1}}\right) \ldots E^{0_{k-1}}\left(\mu_{0_{k-1}}\right)\left\{E^{0_{k}}\left(\mu_{0_{k}}\right) \frac{\hat{h}_{\alpha_{0}}^{a}}{\mu_{0_{k}}-Z_{a}}-\right. \\
& \left.\quad-E^{0_{k} \mid a}\left(\mu_{0_{k}}\right) \sum_{a=1}^{N} \frac{\hat{h}_{\alpha_{0}}^{a}}{\mu_{0_{k}}-Z_{a}}\right\} E^{0_{k+1}}\left(\mu_{0_{k+1}}\right) \ldots E^{0_{p}}\left(\mu_{0_{p}}\right) \psi^{p}= \\
& =\frac{\left(\alpha_{0}, \alpha_{0}\right)^{p}}{2^{p}}\left(\frac{\left(\lambda^{a}, \chi\right)}{\mu_{0_{k}}-Z_{a}} \varphi+\left(\sum_{i>k} \frac{\left(\alpha_{0}, \alpha_{0}\right) / 2}{\mu_{0_{k}}-\mu_{0_{i}}}-\sum_{b=1}^{N} \frac{\left(\lambda^{b}, \chi\right)}{\mu_{0_{k}}-Z_{b}}\right) \varphi_{\mu_{0_{k}}}^{a}\right)- \\
& \quad-\sum_{i>k} \frac{\left(\alpha_{0}, \alpha_{0}\right) / 2}{\mu_{0_{k}}-\mu_{0_{i}}} E^{0_{1}}\left(\mu_{0_{1}}\right) \ldots E^{0_{k} \mid a}\left(\mu_{0_{k}}\right) \ldots E^{0_{i}}\left(\mu_{0_{k}}\right) \ldots E^{0_{p}}\left(\mu_{0_{p}}\right) \psi^{p} .
\end{aligned}
$$

Аналогично с учетом соотношений (26), (44) мы получим

$$
\begin{aligned}
& E^{0_{1}}\left(\mu_{0_{1}}\right) \ldots E^{0_{k-1}}\left(\mu_{0_{k-1}}\right) \times \\
& \quad \times\left\{\frac{\left(\alpha_{0}, \alpha_{0}\right)}{2} \sum_{i<k} \frac{\hat{h}_{\alpha_{0}}^{0_{i}}}{\mu_{0_{k}}-\mu_{0_{i}}} E^{0_{k} \mid a}\left(\mu_{0_{k}}\right)-E^{0_{k} \mid a}\left(\mu_{0_{k}}\right) H_{k}^{0_{k}}\right\} \ldots E^{0_{p}}\left(\mu_{0_{p}}\right) \psi^{p}= \\
& =\frac{\left(\alpha_{0}, \alpha_{0}\right)^{p}}{2^{p}}\left(\sum_{j>k} \frac{\left(\alpha_{0}, \alpha_{0}\right) / 2}{\mu_{0_{k}}-\mu_{0_{j}}} \frac{\mu_{0_{j}}-Z_{a}}{\mu_{0_{k}}-Z_{a}} \varphi_{\mu_{0_{j}}}^{a}+\sum_{i \neq k} \frac{\left(\chi, e_{-\alpha_{0}} \chi\right)}{\mu_{0_{k}}-\mu_{0_{i}}} \varphi_{\mu_{0_{k}}}^{a}\right)+ \\
& \quad+E^{0_{1}}\left(\mu_{0_{1}}\right) \ldots E^{0_{k} \mid a}\left(\mu_{0_{k}}\right) \ldots E^{0_{p}}\left(\mu_{0_{p}}\right) H_{p}^{0_{k}} \psi^{p} .
\end{aligned}
$$

Согласно определению векторов $\psi^{p}$ и $\psi^{p \mid \rho}$ имеем

$$
P^{0_{k}} \psi^{p}=0, \quad P^{0_{k}} \psi^{p \mid \rho}=0,
$$

так что вклад последних членов из уравнения (41) в правой части (42) обрашается в нуль.

Теперь для окончания доказательства равенств (6)-(9) остается сложить соотношения $(37),(38)$ и (43)-(46) в соответствии с (41) и (42).

В заключение скажем несколько слов об оставшихся простых комплексных алгебрах Ли $E_{8}, F_{4}, G_{2}$.

Если в множестве $A$, определяюшем $\varphi$ в (5), хотя бы один простой корень имеет кратность 1, то, обозначая этот корень через $\alpha_{0}$, мы придем к соотношениям $(18),(20)$ и $(21)$. 
Кроме того, последние слагаемые в правых частях равенств (37), (38) обращаются в нуль, и приведеннное доказательство полностью подходит и к этому случаю. Однако в случае произвольного множества $A$ соотношения (24)-(26) не выполняются, и наше доказательство не имеет места.

ПРИЛОЖЕНИЕ

Пусть $\left\{A_{i}\right\}_{i=1}^{n}$ - операторы в векторном пространстве $V$. Сопоставим каждому оператору комплексньй параметр $\mu_{i} \in \mathbf{C}: A_{i} \leftrightarrow \mu_{i}$. Пары $\left(A_{i} ; \mu_{i}\right)$ составляют множество $A=\left\{\left(A_{i} ; \mu_{i}\right), i=1, \ldots, n\right\}$.

УТВЕРЖДЕнИЕ:

$$
\begin{aligned}
& \sum_{p \in S_{|K|}} \frac{A_{p(1)} \ldots A_{p|K|}}{\left(\mu_{p(1)}-\mu_{p(2)}\right) \ldots\left(\mu_{p|K|}-Z\right)}= \\
& =\sum_{\substack{\left(K_{1}, K_{2}\right), K_{1} \cap K_{2}=\varnothing \\
\bigcup_{a} K_{a}=A /\left[A_{n} ; \mu_{n}\right]}} \sum_{q \in S_{\left|K_{1}\right|}} \sum_{r \in S_{\left|K_{2}\right|}} \frac{\left[A_{q(1)}^{K_{1}},\left[\ldots\left[A_{q\left|K_{1}\right|}^{K_{1}}, A_{n}\right] \ldots\right]\right.}{\left(\mu_{q(1)}^{K_{1}}-\mu_{q(2)}^{K_{1}}\right) \ldots\left(\mu_{q\left|K_{1}\right|}^{K_{1}}-\mu_{n}\right)\left(\mu_{n}-Z\right)} \times \\
& \quad \times \frac{A_{r(1)}^{K_{2}} \ldots A_{r\left(\left|K_{2}\right|\right)}^{K_{2}}}{\left(\mu_{r(1)}^{K_{2}}-\mu_{r(2)}^{K^{2}}\right) \ldots\left(\mu_{r\left(\left|K_{2}\right|\right)}^{K_{2}}-Z\right)}
\end{aligned}
$$

где $K_{1}$ и $K_{2}-$ подмножества $A$ :

$$
K_{i}=\left\{\left(A_{l}^{K_{i}} ; \mu_{i}^{K_{i}}\right) ;\left(A_{l}^{K_{i}} ; \mu_{i}^{K_{i}}\right) \equiv\left(A_{j_{l}} ; \mu_{j_{l}}\right) \in A, l=1, \ldots,\left|K_{i}\right| \leq n\right\}
$$

a $S(k)$ - все перестановки чисел $1, \ldots, k$.

Это комбинаторная задача, которая решается методом математической индукции. В случае $n=1$ соотношение (П.1) очевидно. Мы допускаем, что оно имеет место при $n=m$, и будем доказывать его корректность при $n=m+1$. Обозначим

$$
\begin{aligned}
& f_{1}^{n}\{A\}(Z)=f_{1}^{n}\left\{\left(A_{1} ; \mu_{1}\right) \ldots\left(A_{n} ; \mu_{n}\right)\right\}(Z)=\sum_{p \in S_{|K|}} \frac{A_{p(1)} \ldots A_{p|K|}}{\left(\mu_{p(1)}-\mu_{p(2)}\right) \ldots\left(\mu_{p|K|}-Z\right)}, \\
& f_{2}^{n}\{A\}(Z)=f_{2}^{n}\left\{\left(A_{1} ; \mu_{1}\right) \ldots\left(A_{n-1} ; \mu_{n-1}\right) \|\left(A_{n} ; \mu_{n}\right)\right\}(Z)= \\
& =\sum_{\substack{\left(K_{1}, K_{2}\right), K_{1} \cap K_{2}=\varnothing \\
\bigcup_{a} K_{a}=A /\left[A_{n} ; \mu_{n}\right]}} \sum_{q \in S_{\left|K_{1}\right|}} \sum_{r \in S_{\left|K_{2}\right|}} \frac{\left[A_{q(1)}^{K_{1}}, \ldots\left[A_{q\left|K_{1}\right|}^{K_{1}}, A_{n}\right] \ldots\right]}{\left(\mu_{q(1)}^{K_{1}}-\mu_{q(2)}^{K_{1}}\right) \ldots\left(\mu_{q\left|K_{1}\right|}^{K_{1}}-\mu_{n}\right)\left(\mu_{n}-Z\right)} \times \\
& \quad \times \frac{A_{r(1)}^{K_{2}} \ldots A_{r\left(\left|K_{2}\right|\right)}^{K_{2}}}{\left(\mu_{r(1)}^{K_{2}}-\mu_{r(2)}^{K^{2}}\right) \ldots\left(\mu_{p\left(\left|K_{2}\right|\right)}^{K_{2}}-Z\right)} .
\end{aligned}
$$

Как функции от комплексного переменного $f_{1}^{m+1}(Z)$ и $f_{2}^{m+1}(Z)$ имеют сингулярности только в точках $\left\{\mu_{i}\right\}_{i=1}^{m+1}$. В остальных точках зависимость $f_{1}^{m+1}$ и $f_{2}^{m+1}$ от $\mathrm{Z}$ аналитическая, причем

$$
\lim _{|Z| \rightarrow \infty} f_{1}^{m+1}=\lim _{|Z| \rightarrow \infty} f_{2}^{m+1}=0 .
$$


Из (П.2) и теоремы Лиувилля следует, что для доказательства предложения достаточно показать, что

$$
\operatorname{res}_{Z=\mu_{i}} f_{1}^{m+1}=\operatorname{res}_{Z=\mu_{i}} f_{2}^{m+1}, \quad i=1, \ldots,(m+1) .
$$

При $i=1, \ldots, m$ из предположения индукции имеем

$$
\begin{aligned}
\operatorname{res}_{Z} & =\mu_{i} f_{2}^{m+1}\left\{\left(A_{1} ; \mu_{1}\right) \ldots\left(A_{m} ; \mu_{m}\right) \|\left(A_{m+1} ; \mu_{m+1}\right)\right\}(Z)= \\
& =f_{2}^{m}\left\{\left(A_{1} ; \mu_{1}\right) \ldots\left(\widehat{A_{i} ; \mu_{i}}\right) \ldots\left(A_{m} ; \mu_{m}\right) \|\left(A_{m+1} ; \mu_{m+1}\right)\right\}\left(\mu_{i}\right) \times A_{i}= \\
& =f_{1}^{m}\left\{\left(A_{1} ; \mu_{1}\right) \ldots\left(\widehat{A_{i} ; \mu_{i}}\right) \ldots\left(A_{m+1} ; \mu_{m+1}\right)\right\}\left(\mu_{i}\right) \times A_{i}=\operatorname{res}_{Z=\mu_{i}} f_{1}^{m+1}(Z),
\end{aligned}
$$

где “へ” означает, что соответствующий член отпущен. Чтобы полностью иметь (П.3), остается рассмотреть случай $i=m+1$ :

$$
\begin{aligned}
& \operatorname{res}_{Z=\mu_{m+1}} f_{1}^{m+1}\left\{\left(A_{1} ; \mu_{1}\right) \ldots\left(A_{m+1} ; \mu_{m+1}\right)\right\}(Z)= \\
& =f_{1}^{m}\left\{\left(A_{1} ; \mu_{1}\right) \ldots\left(A_{m} ; \mu_{m}\right)\right\}\left(\mu_{m+1}\right) \times A_{m+1}= \\
& =A_{m+1} \times f_{1}^{m}\left\{\left(A_{1} ; \mu_{1}\right) \ldots\left(A_{m} ; \mu_{m}\right)\right\}\left(\mu_{m+1}\right)+ \\
& +\left[f_{1}^{m}\left\{\left(A_{1} ; \mu_{1}\right) \ldots\left(A_{m} ; \mu_{m}\right)\right\}, A_{m+1}\right]= \\
& =A_{m+1} \times f_{1}^{m}\left\{\left(A_{1} ; \mu_{1}\right) \ldots\left(A_{m} ; \mu_{m}\right)\right\}\left(\mu_{m+1}\right)+ \\
& +\sum_{i=1}^{m} f_{1}^{m}\left\{\left(A_{1} ; \mu_{1}\right) \ldots\left(\left[A_{i}, A_{m+1}\right]\right) \ldots\left(A_{m} ; \mu_{m}\right)\right\}\left(\mu_{m+1}\right)= \\
& =A_{m+1} \times f_{1}^{m}\left\{\left(A_{1} ; \mu_{1}\right) \ldots\left(A_{m} ; \mu_{m}\right)\right\}\left(\mu_{m+1}\right)+ \\
& +\sum_{i=1}^{m} f_{2}^{m}\left\{\left(A_{1} ; \mu_{1}\right) \ldots\left(\widehat{A_{i} ; \mu_{i}}\right) \ldots\left(A_{m} ; \mu_{m}\right) \|\left(\left[A_{i}, A_{m+1}\right] ; \mu_{i}\right)\right\}\left(\mu_{m+1}\right)= \\
& =A_{m+1} \times f_{1}^{m}\left\{\left(A_{1} ; \mu_{1}\right) \ldots\left(A_{m} ; \mu_{m}\right)\right\}\left(\mu_{m+1}\right)+ \\
& +\sum_{i=1}^{m} \sum_{\substack{\left(K_{1}, K_{2}\right), K_{1} \cap K_{2}=\varnothing}} \sum_{q \in S_{\left|K_{1}\right|}} \sum_{r \in S_{\left|K_{2}\right|}} \frac{\left[A_{q(1)}^{K_{1}},\left[\ldots\left[A_{q\left|K_{1}\right|}^{K_{1}},\left[A_{i}, A_{m+1}\right]\right] \ldots\right]\right.}{\left(\mu_{q(1)}^{K_{1}}-\mu_{q(2)}^{K_{1}}\right) \ldots\left(\mu_{q\left|K_{1}\right|}^{K_{1}}-\mu_{i}\right)\left(\mu_{i}-\mu_{m+1}\right)} \times \\
& \cup_{a} K_{a}=A \backslash\left(A_{i} \cup A_{m+1}\right) \\
& \times \frac{A_{r(1)}^{K_{2}} \ldots A_{r\left(\left|K_{2}\right|\right)}^{K_{2}}}{\left(\mu_{r(1)}^{K_{2}}-\mu_{r(2)}^{K^{2}}\right) \ldots\left(\mu_{p\left(\left|K_{2}\right|\right)}^{K_{2}}-\mu_{m+1}\right)} .
\end{aligned}
$$

Последнее равенство следует из предположения индукции

$$
\begin{aligned}
\operatorname{res} Z=\mu_{m+1} & f_{2}^{m+1}\left\{\left(A_{1} ; \mu_{1}\right) \ldots\left(A_{m} ; \mu_{m} \|\left(A_{m+1} ; \mu_{m+1}\right)\right\}(Z)=\right. \\
= & \sum_{\substack{\left(K_{1}, K_{2}\right), K_{1} \cap K_{2}=\varnothing \\
\cup_{a} K_{a}=A \backslash\left[A_{m+1} ; \mu_{m+1}\right]}} \sum_{q \in S_{\left|K_{1}\right|}} \sum_{r \in S_{\left|K_{2}\right|}} \frac{\left[A_{q(1)}^{K_{1}},\left[\ldots\left[A_{q\left|K_{1}\right|}^{K_{1}}, A_{m+1}\right] \ldots\right]\right.}{\left(\mu_{q(1)}^{K_{1}}-\mu_{q(2)}^{K_{1}}\right) \ldots\left(\mu_{q\left|K_{1}\right|}^{K_{1}}-\mu_{m+1}\right)} \times \\
& \times \frac{A_{r(1)}^{K_{2}} \ldots A_{r\left(\left|K_{2}\right|\right)}^{K_{2}}}{\left(\mu_{r(1)}^{K_{2}}-\mu_{r(2)}^{K^{2}}\right) \ldots\left(\mu_{r\left(\left|K_{2}\right|\right)}^{K_{2}}-\mu_{m+1}\right)}
\end{aligned}
$$


Нетрудно заметить, что любой член суммы (П.4) можно найти в правой части (П.5). Действительно, первый член в правой части (П.4) равен подсумме (П.5) с $K_{1}=\varnothing$ в силу того, что в этом случае

$$
\sum_{\substack{\left(K_{1}, K_{2}\right), K_{1} \cap K_{2}=\varnothing \\ \cup_{a} K_{a}=A \backslash\left[A_{m+1} ; \mu_{m+1}\right]}} \sum_{q \in S_{\left|K_{1}\right|}} \sum_{r \in S_{\left|K_{2}\right|}}=A_{m+1} \times \sum_{p \in S(m)} .
$$

Что же касается слагаемого из последней подсуммы (П.4) с конкретным $i$, то его можно найти в (П.5) с $A_{i} \in K_{1}, A_{q\left|K_{1}\right|}^{K_{1}}=A_{i}$. Из вьшесказанного и из того, что число членов суммы (П.4) $m !+\sum_{k=1}^{m} m !=(m+1) !$ равно числу членов суммы $(\Pi .5) \sum_{k=0}^{m} C_{k}^{m}(m-$ $k) ! k !=(m+1) !$, следует равенство этих сумм.

Таким образом, мы полностью доказали (П.3), доказывая тем самым исходное утверждение.

Автор благодарен Г. М. Бабуджяну за постановку задачи и Р. Г. Погосяну за многократные полезные обсуждения.

\section{Список литературы}

[1] М. Годен. Волновая функция Бете. М.: Мир, 1987.

[2] C. N. Yang. Phys. Rev. Lett. 1967. V. 19. P. 1312.

[3] B. Sutherland. Phys. Rev. Lett. 1967. V. 20. P. 98

[4] P. P. Kulish, N. Yu. Reshetikhin. J. Phys. A: Math. Gen. 1983. V. 16. P. 591.

[5] M. Karowski, P. Weisz. Nucl. Phys. B. 1978. V. 139. P. 495.

[6] F. A. Smirnov. Formfactors In Completely Integrable Models Of Quantum Field Theory. Singapore: World Scientific Pub. Co., 1990.

[7] H. M. Babujian, R. Flume. Mod. Phys. Lett. A. 1994. V. 9. № 22. P. 2029.

[8] V. G. Knizhnik, A. B. Zamolodchikov. Nucl. Phys. B. 1984. V. 247. P. 83.

[9] H. M. Babujian. Correlation function in WZNW model as a Bethe wave function for the Gaudin magnetics. In: Proc. XXIV Int. Symp. Ahrenshoop, Zeuthen 1990. Preprint YERPHI-1261 (4) -90 .

[10] B. Feigin, E. Frenkel, N. Yu. Reshetikhin. Commun. Math. Phys. 1994. V. 166. P. 27.

[11] Л. А. Тахтаджян, Л. Д. Фаддеев. Гамильтонов подход в теории солитонов. М.: Наука, 1986.

[12] Д. П. Желобенко, А. И. Штерн. Представления групп Ли. М.: Наука, 1983.

Поступила в редакцию 5.II.1997 г.

\section{V.V. Mkhitaryan \\ GAUDIN MAGNET AND OFF MASS SHELL BETHE WAVE FUNCTIONS}

The general Bethe equation (or off-shell Bethe ansatz equation) is proved for the Gaudin magnet in the case of a wide class of complex simple Lie algebras. 